This accepted author manuscript is copyrighted and published by Elsevier. It is posted here by agreement between Elsevier and MTA. The definitive version of the text was subsequently published in [Brain Behavior And Immunity, 38: pp. 25-35, 2014, DOI: 10.1016/j.bbi.2014.01.010]. Available under license CC-BY-NC-ND. 
Revised version Ms. Ref. No.: BBI-D-13-00403

Full length Research report to:

Brain Behavior and Immunity 2014 Named Series on Diet, Inflammation and the Brain Host Editor: Teresa M. Reyes

\title{
The Fractalkine/Cx3CR1 System is Implicated in the Development of Metabolic Visceral Adipose Tissue Inflammation in Obesity
}

\author{
Ágnes Polyák, Szilamér Ferenczi, Ádám Dénes, Zsuzsanna Winkler, Rókus Kriszt, Bernadett \\ Pintér-Kübler and Krisztina J. Kovács \\ Laboratory of Molecular Neuroendocrinology Institute of Experimental Medicine, Budapest, \\ Hungary,
}

Corresponding author:

Krisztina J. Kovács, PhD, DSc.

Laboratory of Molecular Neuroendocrinology

Institute of Experimental Medicine,

Szigony utca 43. Budapest, Hungary,

E-mail: kovacs@koki.hu

Tel: +36-1-210-9952

Fax: +36-1-210-9423 


\begin{abstract}
Diet-induced obesity and related peripheral and central inflammation are major risk factors for metabolic, neurological and psychiatric diseases. The chemokine fractalkine (Cx3CL1) and its receptor $\mathrm{Cx} 3 \mathrm{CR} 1$ play a pivotal role in recruitment, infiltration and proinflammatory polarization of leukocytes and micoglial cells, however, the role of fractalkine signaling in the development of metabolic inflammation is not fully resolved. To address this issue, fractalkine receptor deficient (Cx3CR1 gfp/gfp) mice were exposed to normal or fat-enriched diet (FatED) for 10 weeks and physiological-, metabolic- and immune parameters were compared to those animals in which the fractalkine signaling is maintained by the presence of one functioning allele (Cx3CR1 $+/ g f p)$. Mice with intact fractalkine signaling develop obesity characterized by increased epididymal white fat depots and mild glucose intolerance, recruit leukocytes into the visceral adipose tissue and display increased expression of subset of pro- and anti-inflammatory cytokines when exposed to fat-enriched diet. By contrast, Cx3CR1-deficient (gfp/gfp) mice gain significantly less weight on fat-enriched diet and have smaller amount of white adipose tissue (WAT) in the visceral compartment than heterozygote controls. Furthermore, Cx3CR1 gfp/gfp mice fed a fat-enriched diet do not develop glucose intolerance, recruit proportionally less number of gfp-positive cells and express significantly less MCP-1, IL-1a and TNFa in the WAT than control animals with fat-enriched diet induced obesity. Furthermore, heterozygote obese, but not fractalkine receptor deficient mice express high levels of anti-inflammatory IL-10 and arginase 1 markers in the visceral fat. The effect of fat-enriched diet on cytokine expression pattern was specific for the WAT, as we did not detect significant elevation of interleukin-1, tumor necrosis factor-alpha and monocyte chemoatracting protein (MCP-1) expression in the liver or in the hypothalamus in either genotype. These results highlight the importance of fractalkine signaling in recruitment and polarization of adipose tissue immune cells and identify fractalkine as a target to fight obesity-induced inflammatory complications.
\end{abstract}

Key words: White adipose tissue, Brown adipose tissue, Liver, Hypothalamus, High fat diet, interleukin-1, IL- $1 \alpha$ and $\beta$, IL-6, TNF $\alpha$, MCP-1, HSD1.

\author{
Abbreviations \\ ACTH - adrenocorticotropic hormone \\ ARG1 - arginase 1 \\ ACK - Ammonium-Chloride-Potassium Lysing Buffer \\ ATM - adipose tissue macrophages \\ BAT - brown adipose tissue \\ CORT - corticosterone
}


$\mathrm{CRH}$ - corticotropin-releasing hormone

Cx3CL1 - fractalkine

Cx3CR1 - fractalkine receptor

Dnase - deoxyribonuclease

EDTA - ethylenediaminetetraacetic acid

EWAT - epididymal white adipose tissue

FatED - fat-enriched diet

FKN - fractalkine

GAPDH - glyceraldehyde 3-phosphate dehydrogenase

GFP - green fluorescent protein

GTT - glucose tolerance test

HFD - high fat diet

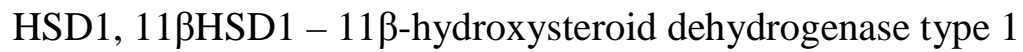

IBAT - interscapular brown adipose tissue

IL-10 - interleukin-10

IL-1a - interleukin-1 alpha

IL-1b - interleukin-1 beta

IL-6 - interleukin-6

MCP-1, CCL2 - monocyte chemotactic protein-1

NALP3 - NACHT, LRR and PYD domains-containing protein 3

ND - normal diet

NLRP3 - NLR family, pyrin domain containing 3

NPY - neuropeptide Y

POMC - pro-opiomelanocortin

RIA - radioimmunoassay 
RT-PCR - real-time polymerase chain reaction

SWAT - subcutaneous white adipose tissue

$\mathrm{TNFa}$ - tumor necrosis factor alpha

UCP1 - uncoupling protein 1

WAT - white adipose tissue 


\section{INTRODUCTION}

Energy metabolism is one of the most rigorously regulated homeostatic functions controlled by central and peripheral mechanisms [1]. During overload of fuels, excess of fat is deposited into the white adipose tissue (WAT) depots that are capable to synthesize and release various endocrine and immune mediators as well $[2 ; 3]$. Visceral WAT from lean animals contains resident population of alternatively activated macrophages, which are characterized by expression of M2 markers such as arginase, F4/80 and CD301 [4]. Expanding WAT in obese subjects attracts various immune cells and release pro-inflammatory cytokines that contribute to the "low grade chronic metabolic inflammation" that represents a significant health burden [5]. Growing evidence implicates that obesity-induced tissue inflammation is not limited to the visceral WAT but also seen in the liver and in the hypothalamus [6]. In either tissue, diet-induced inflammation is always associated with recruitment/proliferation and activation of various immune-competent cells such as monocytes, macrophages and $\mathrm{T}$ cells.

Fractalkine (Cx3CL1, neurotactin) is a chemokine expressed in endothelial cells, vascular smooth muscle cells, hepatocytes, adipocytes and neurons as a transmembrane protein and involved in recruitment and capturing various leukocytes (monocytes, macrophages, microglia) expressing its' cognate receptor, Cx3CR1 [7; 8; 9]. Fractalkine can be released from the cell surface by proteolytic cleavage and might act in paracrine and endocrine manner. Fractalkine is an important regulatory factor of microglia activity in the central nervous system mediating neuroinflammation. However its role in metabolic inflammation in general, and in connecting metabolic and neuroinflammation in particular, remains to be elucidated. It has recently been shown that fractalkine is an adipocytokine in humans [10]. Furthermore, elevated plasma fractalkine levels were detected in patients with type 2 diabetes and single nucleotide polymorphism (rs3732378) in Cx3CR1 was associated with changes in adipose markers and metabolic parameters [10].

To address the role of fractalkine signaling in the recruitment and activation of immune responses in key central (hypothalamus) and peripheral (visceral WAT and liver) structures, we have compared fat-enriched diet induced changes in metabolic physiology and tissue-specific expression of proinflammatory cytokines of mice in which $\mathrm{Cx} 3 \mathrm{CR} 1$ receptors were compromised. 


\section{MATERIALS and METHODS}

\subsection{Animals and diet}

Experiments were performed in male Cx3CR1 +/gfp, and Cx3CR1 gfp/gfp mice [11]. Animals were obtained from the European Mouse Mutant Archive (EMMA cx3cr1tm1Litt MGI:2670351), backcrossed for more than 10 generations to C57B1/6. The C57B1/6J strain has been shown to be genetically vulnerable to diet-induced obesity [12]. In these mice, the $\mathrm{cx} 3 \mathrm{cr} 1$ gene was replaced by a gfp reporter gene such that heterozygote Cx3CR1+/gfp mice express GFP in cells that retain receptor function, whereas cells in homozygote Cx3CR1gfp/gfp (Cx3CR1-/-, knockout (KO)) mice are labeled with GFP and also lack functional Cx3CR1. Genotype of the animals has been verified by PCR using combination of three different primers as described by Jung et al. [11].

Animals were housed in groups of 4-5/cage at the minimal disease (MD) level of the Medical Gene Technology Unit of the Institute, had free access to food and water and were maintained under controlled conditions: temperature, $21^{\circ} \mathrm{C} \pm 1^{\circ} \mathrm{C}$; humidity, $65 \%$; light-dark cycle, 12 -h light/12-h dark cycle, lights on at 07:00. At 35 days of age both Cx3CR1 +/gfp $(n=8)$ and Cx3CR1 gfp/gfp $(n=10)$ mice were randomly distributed into two equal groups. The first group, normal diet (ND), received standard chow [VRF1 (P), Special Diets Services (SDS), Witham, Essex, UK calory content: $14,24 \mathrm{KJ} / \mathrm{g}$ ]. In the second group received fat-enriched diet (FatED), by providing a 2:1 mixture of standard chow and lard (Spar Budget, Budapest, Hungary). The calory content of this mixture is $22,02 \mathrm{KJ} / \mathrm{g}$ ). All procedures were conducted in accordance with the guidelines set by the European Communities Council Directive (86/609 EEC) and approved by the Institutional Animal Care and Use Committee of the Institute of Experimental Medicine (permit number: 22.1/3347/003/2007).

\subsection{Experimental design}

Mice were fed with normal diet (ND) or fat enriched diet (FatED) for 10 weeks, body weight and food consumption was measured weekly. In the 10th week glucose tolerance test (GTT) was performed after overnight fasting. Two days after the GTT, mice were decapitated, trunk blood was collected on EDTA, and the plasma stored at $-20^{\circ} \mathrm{C}$ until assay. Brain, liver, visceral- and, subcutaneous white adipose tissue pads and interscapular brown adipose tissue were collected, sampled and stored at $-70^{\circ} \mathrm{C}$ for RT-PCR, or fixed in $4 \%$ buffered paraformaldehyde for histology.

\subsection{Glucose tolerance test}

Mice were fasted overnight $(15 \mathrm{~h})$ and then injected intraperitoneally with $2 \mathrm{mg} / \mathrm{g}$ of body weight D-glucose (20\% stock solution in saline). Blood glucose was measured from tail vein by DCont Personal Blood Glucose Meter (77 Elektronika Kft. Hungary) at 0 min (just before glucose injection) and at 15-, 30-, 60-, 90- and 120-min intervals after the glucose load. 


\subsection{Hormone and Cytokine Measurements}

Plasma adrenocorticotrophic hormone (ACTH) and corticosterone (CORT) concentrations were measured by radioimmunoassay (RIA) as described. ACTH RIA was developed in our laboratory [13] using an antibody (\#8514) raised against the mid-portion of human ACTH1-39. The test uses $50 \mu 1$ of plasma per determination, has a lower limit of sensitivity of $0.1 \mathrm{fmol} / \mathrm{ml}$, and the average intra-and inter-assay coefficients of variation are $4.8 \%$ and $7.0 \%$ respectively. Plasma corticosterone has been measured by a direct RIA without extraction as described [14]. The intra and interassay \%CVs in this assay are 12.3 and 15.3 respectively. Plasma cytokine levels were measured with ELISA using DuoSet ELISA kits for IL-1 $\alpha$, IL-1 $\beta$ and IL-6 (R\&DSystems, Minneapolis, MN, USA) according to the manufacturer's protocol. Plasma samples were loaded at 1:5 dilution, the lowest detectable level was $3.9 \mathrm{pg} / \mathrm{ml}$.

\subsection{Histology}

Tissues were fixed by immersion in $4 \%$ paraformaldehyde in $0.1 \mathrm{M}$ phosphate buffer, $\mathrm{pH} 7.4$ (PB) for 3 days. Subsequently, they were stored in $1 \%$ paraformaldehyde in $0.1 \mathrm{M} \mathrm{PB}$ at $4{ }^{\circ} \mathrm{C}$. Tissues were paraffin-embedded, sectioned and stained with hematoxylin-eosin (H\&E). Slides were digitalized with Pannoramic Digital Slide Scanner (3DHISTECH Kft., Hungary) and cell areas were analyzed with ImageJ software (NIH, USA).

Leukocytes in the epididymal WAT were identified directly on the sections and counted under 60x magnification, in 5 random fields/animal on hematoxylin-eosin stained material.

\subsection{Genomic DNA isolation and gfp copy number assay (CNA)}

Genomic DNA has been isolated from tail and fat tissue samples according to standard protocol. gfp copy number has been determined by TaqMan® Copy Number Assay (Assay ID:

Mr00660654_cn Reporter/Quencher: FAM/MGB-NFQ); Life Technologies Foster City, CA, USA).

\subsection{Gene expression analysis by quantitative real-time PCR}

Total RNA was isolated from epididymal white adipose tissue (EWAT) samples with QIAGEN RNeasyMiniKit (Qiagen, Valencia, CA, USA) according the manufacturer's instruction. To eliminate genomic DNA contamination, DNase I (Fermentas) treatment was used. Sample quality control and the quantitative analysis were carried out by NanoDrop (Thermo Scientific). Amplification was not detected in the RT-minus controls. cDNA synthesis was performed with the High Capacity cDNA Reverse Transcription Kit (Applied Biosystems, Foster City, CA, USA). The designed primers (Invitrogen) were used in the real-time PCR reaction with Power SYBR Green PCR master mix (Applied Biosystems, Foster City, CA, USA) on ABI StepOne instrument. The gene expression was analyzed by ABI StepOne2.0program. The amplicon was 
tested by Melt Curve Analysis on ABI StepOne instrument. Experiments were normalized to

Glyceraldehyde 3-phosphate dehydrogenase (GAPDH) expression.

\subsection{Primer design}

Primers used for the comparative CT (threshold cycle) experiments were designed by the Primer Express 3.0 program. Primer sequences were the following:

GAPDH:f: TGA CGT GCC GCC TGG AGA AA r: AGT GTA GCC CAA GAT GCC CTT CAG

IL1a: f: CCA TAA CCC ATG ATC TGG AAG AG r: GCT TCA TCA GTT TGT ATC TCA AAT CAC

IL1b: f: CTC GTG GTG TCG GAC CCA TAT GA r: TGA GGC CCA AGG CCA CAG GT

IL6: f: TCC GGA GAG GAG ACT TCA CA r: TGC AAG TGC ATC ATC GTT GT

TNFa: f: CAG CCG ATG GGT TGT ACC TT r: GGC AGC CTT GTG CCT TGA

NALP3: f: CAG AGC CTA CAG TTG GGT GAA r: ACG CCT ACC AGG AAA TCT CG

IL-10: f:AGT GAG AAG CTG AAG ACC CTC AGG r: TTC ATG GCC TTG TAG ACA CCT TGG T

ARG1:f: GTC TGG CAG TTG GAA GCA TCT r: GCA TCC ACC CAA ATG ACA CA

MCP-1:f: CCAGCACCAGCACCAGCCAA r: TGGATGCTCCAGCCGGCAAC

FKN: f: CCG CGT TCT TCC ATT TGT GT r: GGT CAT CTT GTC GCA CAT GATT

GFP: f: GGA CGA CGG CAA CTA CAA GA r: AAG TCG ATG CCC TTC AGC TC

UCP1: f: GGTCAAGATCTTCTCAGCCG r: AGGCAGACCGCTGTACAGTT

HSD1: f: CCTCCATGGCTGGGAAAAT r: AAAGAACCCATCCAGAGCAAAC

POMC:f: TGCTTCAGACCTCCATAGATGTGT r: GGATGCAAGCCAGCAGGTT

NPY: f: CAGATACTACTCCGCTCTGCGACACTACAT r: TTCCTTCATTAAGAGGTCTGAAATCAGTGT

CRH: f: CGCAGCCCTTGAATTTCTTG r: CCCAGGCGGAGGAAGTATTCTT 


\subsection{Normalized $g f p$ expression}

Because the coding region of the $c \times 3 c r 1$ gene has been replaced by $g f p$ in our experimental animals [11], we relied on gfp expression to estimate and compare Cx3CR1 gene expression in Cx3CR1 homo-(gfp/gfp) and heterozygote (+/gfp) animals. To resolve different gfp copy numbers in homo- and heterozygotes we have calculated normalized gfp expression using the following formula: RQ / CN, where RQ is the relative quantity of gfp from real time qPCR measurement and $\mathrm{CN}$ is the gfp copy number determined from genomic DNA by Taqman Copy Number Assay.

\subsection{Stromal Vascular Fraction preparation and Flow Cytometry}

Epididymal white fat pads have been dissected from mice after decapitation. Fat tissue was minced into small pieces and digested in Krebs-Ringer HEPES (KRH) buffer $(\mathrm{pH}=7.4)$ containing $1 \%$ BSA and $1 \mathrm{mg} / \mathrm{ml}$ Collagenase (SIGMA C9891) at $37^{\circ} \mathrm{C}$ for $30 \mathrm{~min}$. in shaking bath and then filtered through a $40 \mu \mathrm{m}$ mesh. The cell suspension was centrifuged at 500x g for $10 \mathrm{~min}$. to separate floating adipocytes and stromal vascular cell fraction (SVF) in the pellet. SVF was resuspended in KRH-BSA buffer. Following lysis of red blood cells with ACK solution and $\mathrm{Fc}$ receptor blockade (anti-mouse CD16/CD32, clone 93, eBioscience), cells were labelled with cocktails of selected antibodies: MHCII-APC, Ly6c-PE-Cy7, CD115-APC (eBioscience) and F4/80-PE (Serotec). Specificity of antibodies has been assessed by eBioscience and Serotec, respectively. Cells were acquired on a FACSAriaII flow cytometer (BD Biosciences, US) and data were analyzed using FACS Diva software (BD Biosciences).

\subsection{Statistical analysis}

The results are shown as means \pm SEM. Statistical analysis was performed by factorial ANOVA with Newman-Keuls post-hoc test in Statistica 11 (StatSoft Inc.). Flow cytometric data were analyzed by two-way ANOVA followed by Sidak's multiple comparison test (GraphPad Prism); In all cases $\mathrm{p}<0.05$ was considered significant. 


\section{RESULTS}

\subsection{Body weight change and food consumption}

During the first six weeks of diet, there was no significant difference in body weight. From the $7^{\text {th }}$ to the $10^{\text {th }}$ week, FatED fed mice gained more weight $(\mathrm{F}(1,14)=5.44, \mathrm{p}<0.05 ; \mathrm{F}(1,14)=$ 5.93, $\mathrm{p}<0.05 ; \mathrm{F}(1,14)=14.74, \mathrm{p}<0.01 ; \mathrm{F}(1,14)=18.69, \mathrm{p}<0.001$ respectively $)$. Post-hoc analysis revealed that only weight gain of $+/$ gfp FatED mice differed significantly from the normal diet fed group. There were no differences between Cx3CR1 gfp/gfp FatED, Cx3CR1 gfp/gfp ND and Cx3CR1 +/gfp ND groups (Fig. 1A).

FatED fed mice consumed less food $(\mathrm{F}(1,36)=39.00, \mathrm{p}<0.001)$ than mice on normal diet, there was no difference between genotypes (Fig. 1B). In spite of the differences in food intake, average caloric intake was significantly higher in FatED animals than those on ND (Fig.1C).

3.2 Organ weights FatED fed mice had larger epididymal white adipose tissue (EWAT), interscapular brown adipose tissue (IBAT) fat pads, and thymus $(\mathrm{F}(1,14)=72.17, \mathrm{p}<0.001$; $\mathrm{F}$ $(1,14)=11.50, \mathrm{p}<0.01, \mathrm{~F}(1,14)=5.30, \mathrm{p}<0.05$, respectively) than mice on normal diet. Normalized liver weight was lower in FatED fed mice $(F(1,14)=71.10, p<0.001)$. Post-hoc analysis revealed that EWAT and IBAT fat pads in FatED gfp/gfp mice were significantly smaller than in FatED +/gfp group. There were no differences in IBAT fat pads between +/gfp ND, gfp/gfp ND and gfp/gfp FatED groups (Fig. 2).

\subsection{Blood glucose levels and glucose tolerance test}

FatED fed mice had higher basal blood glucose levels $(\mathrm{F}(1,14)=9.33, \mathrm{p}<0.05)$, but there were no differences in blood glucose levels after overnight fasting (Fig. 3A).

During glucose tolerance test, there were no differences in blood glucose levels after 15, 30, 60 minutes following intraperitoneal glucose load between the groups. After $120 \mathrm{~min}$, blood glucose levels returned to normal in ND fed mice but not in FatED mice $(F(1,14)=8.51, p<0.05)$. As shown in Fig. 3B. post-hoc analysis revealed no differences in +/gfp ND, gfp/gfp ND, and gfp/gfp FatED groups, blood glucose level was higher only in +/gfp FatED group.

\subsection{Hypothalamo-pituitary-adrenocortical (HPA) axis}

Because the neuroendocrine stress axis has been implicated in central metabolic and immune regulation, we assessed corticotropin-releasing hormone (CRH) mRNA levels in the hypothalamus and adrenocorticotropin (ACTH) and corticosterone concentration in the plasma. In the hypothalamus there were no significant differences in CRH mRNA levels, although a trend towards Cx3CR1 gfp/gfp mice having slightly elevated CRH levels was seen (not significant, $(\mathrm{F}(1,14)=3.15, \mathrm{p}=0.09)(\mathrm{Fig} .4 \mathrm{~A}))$. 
Plasma ACTH levels were higher in FatED fed mice than in controls $(\mathrm{F}(1,13)=7.35, \mathrm{p}<0.05)$ (Fig. 4B).

Gfp/gfp mice had higher plasma corticosterone levels compared to heterozygotes $(+/ \mathrm{gfp})(\mathrm{F}$ $(1,12)=5.30, p<0.05)($ Fig. 4 C). The diet was without any significant effect on plasma corticosterone levels.

\subsection{Immune cells and expression inflammation-related markers in response to fat-enriched} diet

\subsubsection{White Adipose Tissue}

Monocytes and macrophages in the epididymal white adipose tissue have been analysed by flow cytometry. CX3CR1 -/- mice fed a chow diet showed significantly reduced macrophage numbers overall (Fig. 5, P<0.01, two-way ANOVA), which was most apparent in the F4/80+ MHCIIhigh population ( $\mathrm{P}<0.05$, two way ANOVA followed by Sidak's multiple comparison test) compared to Cx3CR1 +/- animals. Numbers of Ly6chigh CD115+ or Ly6clow cells were not different between genotypes (not shown).Histology: FatED feeding resulted in larger adipose cell size in eWAT and subcutaneous white adipose tissue $(\mathrm{sWAT})(\mathrm{F}(1,1716)=1403.58, \mathrm{p}<0.001, \mathrm{~F}$ $(1,2546)=1099.63, \mathrm{p}<0.001$, respectively $)$. Adipose cells both in eWAT and sWAT were smaller in FatED gfp/gfp mice than in FatED +/gfp mice (Fig. 6).

10 week long FatED resulted in an increase of the number of leukocytes found in the epididymal adipose tissue of Cx3CR1 +/gfp mice (Fig. 7A).Leukocytes in obese +/gfp animals often formed crown like structures (CLS) around adipose cells in the epididymal WAT, indicative of engulfment of damaged adipocytes by immune cells. The frequency of CLS was dramatically increased in obese Cx3CR1 +/gfp mice compared either to Cx3CR1 gfp/gfp mice kept on FatED or to lean mice from both genotypes (Fig 7B,C).

To further confirm that Cx3CR1 plays a key role in recruitment and/or activation of cells in the adipose tissue, liver and hypothalamus in response to fat-enriched diet, we took advantage of gfp expression in these cells and analyzed normalized gfp mRNA tissue levels. Normalized expression of gfp was elevated in FatED fed mice $(F(1,13)=11.31, p<0.01)$. FatED Cx3CR1 gfp/gfp mice had lower gfp mRNA level than +/gfp mice, although the difference was not significant ( $\mathrm{p}=0.06)$ (Fig. 8A).

MCP-1 and FKN (Cx3CL1) are chemokines that play important role in recruitment, infiltration and activation of monocytes into various endangered tissues. MCP-1 and fractalkine mRNA levels were elevated in FatED fed mice $(F(1,13)=31.25$, $p<0.001, F(1,12)=27.53$, $p<0.001$ respectively). As shown in Fig. 8C MCP-1 mRNA level was 21,5 fold higher in +/gfp FatED group, whereas it was only 5,3 fold higher in gfp/gfp FatED group. There were no differences in FKN levels between genotypes (Fig. 8B) suggesting that absence of the cognate receptor does not affect the expression of the ligand in the white adipose tissue.

M1 markers: 
Among the proinflammatory cytokines, we compared mRNA levels of interleukin1 alpha, beta (IL-1a and b); interleukin 6 (IL-6) and tumor necrosis factor alpha (TNFa) in various tissues from mice kept on normal or on FatED.

As shown in Fig. 8 E-H, FatED increased IL-1a, IL1-b, IL-6, and TNFa mRNA levels (F $(1,13)$ $=29.80, \mathrm{p}<0.001, \mathrm{~F}(1,13)=9.17, \mathrm{p}<0.01, \mathrm{~F}(1,13)=6.42, \mathrm{p}<0.05, \mathrm{~F}(1,13)=79.44, \mathrm{p}<$ 0.001 , respectively). In gfp/gfp FatED mice IL-1a, and TNFa levels were significantly lower than in +/gfp fat dieted group. IL6 mRNA level was lower in gfp/gfp mice $(\mathrm{F}(1,12)=4.71, \mathrm{p}<$ $0.05)$.

Because NALP3 inflammasome is critical in processing pre-proIL-1 into releasable form, we checked if Nod-like receptor (NLR) protein (Nalp3) expression changed in response to fat diet and/or in the absence of fractalkine signaling. As shown in Fig. 8K, fat-enriched diet resulted in elevation of NALP3 mRNA levels in the visceral WAT, $[\mathrm{F}(1,12)=41.15, \mathrm{p}<0.001]$. It has also been revealed that fat-enriched diet induced increase in Nalp3 mRNA was significantly lower in fractalkine receptor deficient mice than that of Cx3CR1 +/gfp animals.

M2 markers:

Alternatively activated macrophages (M2) express set of anti-inflammatory cytokines and mediators involved in tissue restoration. To address changes in select M2 markers during fatenriched dieting, we measured mRNA levels of IL-10 and arginase 1 in the epididymal white fat depots. FatED increased IL-10 and Arg1 mRNA levels $(F(1,12)=21.08, p<0.001, F(1,12)=$ 25.68, p < 0.001). In gfp/gfp fractalkin receptor knockout mice, IL-10 (Fig. 8I) and Arg1 (Fig. $8 \mathrm{~J}$ ) elevation was significantly lower than in +/gfp animals following fat-enriched diet. Increased levels of 11bHSD1 mRNA were found in the epididymal WAT in both genotypes (F $(1,14)=6.92, p<0.05)($ Fig. $8 D)$ as a response to FatED.

\subsubsection{Brown Adipose Tissue}

Reduced weight gain in gfp/gfp mice during FatED raised the possibility that the energy expenditure is different between the genotypes. To this end, we have measured expression of uncoupling protein (UCP-1) mRNA levels in the brown adipose tissue, which is responsible for non-shivering thermogenesis in rodents. As expected, elevated relative levels of UCP mRNA were detected in all animals exposed to FatED, however there was no significant difference between the genotypes $(\mathrm{F}(1,12)=67.57, \mathrm{p}<0.001)$ (Fig. $8 \mathrm{~L})$.

\subsubsection{Liver}

To investigate the effect of FatED on the liver, we have measured relative quantities of IL-1a and b, IL-6, TNFa and MCP-1 mRNA in liver samples of mice from both genotypes after exposure either normal or FatED. As shown on Figure 9, high fat intake increased IL-6 mRNA levels in $+/$ gfp animals $(\mathrm{F}(1,12)=7.85, \mathrm{p}<0.05)$ but not in gfp/gfp mice.. Normalized gfp mRNA levels, IL-1a,b, TNFa and MCP-1 mRNA relative quantities were not different (Fig.9)..

\subsubsection{Hypothalamus}


Neuroinflammation in metabolic-related cell groups of the medial basal hypothalamus has been recently detected in high fat fed rodents and obese humans [6]. Normalized gfp mRNA levels in our samples showed significant genotype, treatment effect, and genotype*treatment interaction: $(\mathrm{F}(1,14)=12,73, \mathrm{p}<0.01 ; \mathrm{F}(1,14)=4.70, \mathrm{p}<0.05 ; \mathrm{F}(1,14)=16.41, \mathrm{p}<0.01$, respectively $)$. Post-hoc analysis revealed increased gfp mRNA level in FatED fed Cx3CR1 +/gfp mice, but not in FatED Cx3CR1 gfp/gfp mice (Fig. 10A).

Relative MCP-1 mRNA levels were lower in Cx3CR1 gfp/gfp mice $(\mathrm{F}(1,14)=4,77, \mathrm{p}<0.05)$ than in the controls (Fig. 10B).

There were no significant differences in proinflammatory cytokine mRNA levels (IL-1a, IL-1b, IL-6 and TNFa) (Fig. 10E-H). Although there was a trend towards a decrease in IL-6 mRNA level in Cx3CR1 gfp/gfp group, it was not statistically significant $(F(1,14)=3,87, p=0.07)$. Among the hypothalamic metabolic-related neuropeptides, FatED feeding decreased the level of orexigenic NPY mRNA level $(\mathrm{F}(1,13)=16.63$, p <0.01) (Fig. 10C) but did not alter anorexigenic POMC mRNA (Fig. 10D).

\subsubsection{Plasma cytokine concentrations}

Plasma IL-1b showed an increase in response to FatED overall (two-way ANOVA, F $(1,12)$ $=17,35, \mathrm{P}=0.013)$. However, post hoc comparison revealed that only $+/$ gfp mice fed a fatenriched diet showed significant increase in plasma IL-1b compared to normal diet, but not gfp/gfp animals ( $\mathrm{P}<0.01$, two-way ANOVA followed by Sidak's multiple comparison). Plasma IL-1a and IL-6 levels were not different in any experimental groups (Figure 11). 


\section{DISCUSSION}

Here we provide evidence that fractalkine receptor deficient mice are protected against the development of fat-enriched diet-induced obesity and metabolic WAT inflammation.

Fractalkine signaling in the visceral WAT promotes recruitment and/or activation of cells of monocytic origin and contributes to activation of pro- and anti-inflammatory cytokines. Symptoms of metabolic inflammation at 10 weeks on fat enriched diet are limited to the visceral adipose tissue, without significant proinflammatory changes in the liver and hypothalamus. Although elevated plasma IL-1b and hepatic IL-6 levels in +/gfp mice are indicative of systemic inflammation following fat-enriched diet, these changes are insufficient to upregulate hypothalamic cytokines and CRH.

High fat diet is a major environmental factor that triggers obesity both in humans and in rodents [15]. There are several diets and paradigms to induce obesity. Among these, we used fatenriched food which has been shown to be a relevant trigger for obesity and related pathologies $[16 ; 17 ; 18 ; 19]$. To exclude any food preference, a mixture of chow and lard was given. Although mice kept on excess fat significantly decreased total gram intake of calorically dense diet to compensate caloric consumption, their caloric intake was significantly higher than those on normal diet. Fat-enriched diet resulted in a decrease of the orexigenic neuropeptide, neuropeptide Y (NPY) mRNA levels in hypothalamus and an increase of uncoupling protein (UCP-1) mRNA in the interscapular brown adipose tissue (BAT) samples assayed by real time qPCR. These transcriptional changes can be regarded as compensatory mechanisms to defend body weight. Activation of BAT UCP-1 expression in response to high fat feeding is documented [20]. Although leptin-induced central melanocortin system has long been implicated in regulation of energy homeostasis via activation of BAT thermogenesis [21;22; 23], we did not observe significant changes in hypothalamic POMC expression in mice on fatenriched diet. Intriguingly, decrease in NPY tone onto BAT-related sympathetic preganglionic neurons might be in connection with UCP-1 induction)[24; 25].

Heterozygous (Cx3CR1 +/gfp) FatED animals with intact fractalkine signaling started to gain more weight than controls at 7 weeks, however the difference in body weight became significant after week 8 . This time course is comparable to those obtained after various high fat diets. By contrast, mice - in which fractalkine signaling is compromised -, gain much less weight when on 
fat-enriched diet than heterozygotes, suggesting that Cx3CR1 gfp/gfp mice are somehow resistant to diet-induced obesity.

In heterozygote (Cx3CR1 +/gfp) mice changes in body weight were accompanied with selective increases in epididymal white adipose tissue pads. Notably, epididymal WAT was also enlarged in Cx3CR1 gfp/gfp mice, but the increase was significantly less than that seen in animals with +/gfp genotype. Our results on diet-induced obesity in Cx3CR1 gfp mice differ from other reports on this model. For instance, Morris et al [26] and Lee et al [27] failed to detect differences in HFD induced body weight and adiposity in fractalkine receptor KO mice and controls. These discrepancies might be due to different fat composition (60\% fat for Morris vs. $33 \%$ in this study), choice of control diet, strain differences (Cx3CR1KO for Lee vs. Cx3CR1gfp in our studies), animal care, start and duration (30 weeks for Morris, 24 weeks for Lee and 10 weeks in this study) of the diet.

Activity of the hypothalamo-pituitary-adrenocortical axis in general, and corticosterone plasma levels in particular have been shown to contribute to regulation of abdominal fat deposition [16; 28]. Data on high fat diet-induced corticosterone concentrations are highly controversial: there are reports on increase, decrease or unchanged levels (see [29] for review). Here we found a tendency for FatED-induced adrenocortical hyperactivity in both genotypes. At cellular level, corticosteroid action is dependent on the activity of type1, 11beta-hydroxysteroid dehydrogenase (11ßHSD1) which converts inactive corticosteroids into active corticosterone in mice. In contrast to previous view [30], recent data support the main effect of high fat diet-induced adipose specific 11ßHSD1 downregulation promotes fat accumulation [31]. Furthermore, high local concentration of corticosterone in adipose tissue and/or elevated plasma concentration in Cx3CR1 gfp/gfp mice might reduce macrophage production of proinflammatory cytokines and promote anti-inflammatory responses [32].

Analysis of resident macrophage population in the white adipose tissue of intact animals revealed significantly less macrophages with F4/80+MHCII high phenotype in Cx3CR1 gfp/gfp animals than in Cx3CR1 +6gfp heterozygotes.

Fat-enriched diet resulted in an increase of normalized gfp mRNA expression in the eWAT, liver and hypothalamus in animals with intact fractalkine signaling, indicative of selective recruitment and/or activation of cells expressing Cx3CR1/gfp. Indeed, obesity induces infiltration of macrophages into the epididymal fat, the adipose tissue macrophage content correlates with the 
measures of obesity and cytokines released from these cells contribute to the insulin resistance of the adipocytes [4; 33]. Our histological analysis of the white adipose tissue confirmed these findings by revealing significantly higher number of leukocytes in obese $+/$ gfp animals than in Cx3CR1 gfp/gfp mice on FatED. Among others, chemokines MCP-1 (CCL2) and fractalkine have been identified as important factors, which recruit macrophages to the adipose tissue [34]. Recent results of Shah et al in humans [10] demonstrated that Cx3CL1 is significantly increased in obesity and revealed adipocytes and stromal vascular fraction as source of this adipochemokine that mediates monocyte adhesion to human adipocytes. Here we have confirmed upregulation of fractalkine transcription in the adipose tissue of mice on fat-enriched diet and identified the fractalkine receptor Cx3CR1 as an important mediator of leukocyte/monocyte recruitment into the visceral fat in obesity. This scenario shows similarities with mouse models of atherosclerosis, where disruption of either Cx3CL1 or Cx3CR1 attenuates macrophage trafficking and inflammation $[35 ; 36 ; 37]$. By contrast, Morris et al did not find differences in total adipose tissue macrophages (ATM), in the ratio of type $1(\mathrm{CD} 11 \mathrm{c}(+))$ to type 2 (CD206(+)) ATMs, expression of inflammatory markers, and T-cell content in epididymal fat from obese Cx3CR1(+/gfp) and Cx3CR1(gfp/gfp) mice [26]. Beside the differences in housing and dieting conditions, these discrepancies raise a relevant possibility that cells attracted to the adipose (target) tissue downregulate their special surface markers.

In addition to cell recruitment, increased normalized gfp mRNA might also be held as an indicator of increased activity of cells equipped with the transgene. In support this option, we have revealed accumulation of leukocytes preferentially in the WAT of Cx3CR1 gfp/gfp mice to form "crown like structures" (CLS) around adipocytes. CLS are regarded as a major source of proinflammatory cytokines in the inflammed fat tissue. Indeed, our data demonstrate upregulation of proinflammatory cytokines IL-1a, IL-1b and TNFa expression in the visceral fat of control mice $(\mathrm{Cx} 3 \mathrm{CR} 1+/ \mathrm{gfp})$ in response to fat-enriched diet. This effect was attenuated in Cx3CR1 gfp/gfp mice, supporting the hypothesis that fractalkine signaling is involved in the activation/polarization of adipose tissue macrophages in obesity [4; 19]. However, the specific leukocyte/macrophage population, which contributes to the local elevation proinflammatory cytokines in the fat challenged adipose tissue, remains to be elucidated.

Processing of pro IL-1 $\beta$ by the NLRP3 inflammasome has recently been implicated in obesity [38]. Here we have revealed an increase of NLRP3 mRNA within the epididymal fat pad of 
Cx3CR1 +/gfp mice fed with fat-enriched diet that might contribute to an elevated tissue levels of IL-1 $\beta$. There are several danger-associated signals that might induce assembly of NLRP inflammasome and sterile inflammation of the visceral fat in obese subjects. For instance, ceramide and palmitate have been implicated in activation and /or priming of the inflammasome in adipose tissue and macrophages [39].

It has been shown previously that adipose tissue macrophages in lean animals express markers characteristic of alternatively polarized (M2) macrophages, while high fat diet-induced obesity results in a phenotypic change to the M1 polarization [40].

In addition to proinflammatory cytokines we have demonstrated significant upregulation of two anti-inflammatory markers IL-10 and arginase 1 mRNA in adipose tissue samples from Cx3CR1 +/gfp animals kept on fat-enriched diet, which can be interpreted as a local compensatory mechanism [41; 42]. It should be noted however, that neither baseline expression, nor dietinduced upregulation of IL-10 and arginase 1 transcription is significant in mice with impaired fractalkine signaling.

Our data demonstrate that fat-enriched diet-induced proinflammatory cytokine expression is limited to the visceral adipose tissue, because IL-1a, IL-1b and TNFa mRNA levels did not change in the liver and in the hypothalamic samples. In contrast, Thaler et al [6] reported in rats a HFD-induced bi-phasic hypothalamic inflammation, the first occurs shortly after starting the diet and the second observed in response to chronic HFD exposure. It remains unknown whether the fat-enriched diet is less obesogenic/immunogenic than other high fat diets and/or the length of the exposure is insufficient to induce widespread central inflammation. For instance, ApoE-/mice kept on highly atherogenic cholate/cholesterol rich diet (Paigen) lose weight and develop vascular inflammation, microglial activation, and leukocyte recruitment in the brain, while those mice that are on fatty Western style diet gain weight and show 57\% lower vascular inflammation in the brain. [43], In summary, we report that mice with impaired fractalkine signaling are protected from fat-enriched diet-induced obesity. The mechanism might involve impaired recruitment of leukocytes/monocytes to the visceral fat and altered balance between locally expressed pro-and anti-inflammatory cytokines. These results open the possibility for Cx3CR1 antagonists to fight obesity and metabolic inflammation-related pathologies.

\section{Acknowledgement}


Technical assistance of Ms Julianna Benkö is acknowledged. The work was supported by grants from Hungarian Research Fund OTKA 109622 to KJK; 109744 to SzF and 109743 to ÁD. 


\section{REFERENCES}

[1] M.W. Schwartz, S.C. Woods, D. Porte, Jr., R.J. Seeley, and D.G. Baskin, Central nervous system control of food intake. Nature 404 (2000) 661-71.

[2] R.S. Ahima, and J.S. Flier, Adipose tissue as an endocrine organ. Trends Endocrinol Metab 11 (2000) 327-32.

[3] R.S. Ahima, C.B. Saper, J.S. Flier, and J.K. Elmquist, Leptin regulation of neuroendocrine systems. Front Neuroendocrinol 21 (2000) 263-307.

[4] S.P. Weisberg, D. McCann, M. Desai, M. Rosenbaum, R.L. Leibel, and A.W. Ferrante, Jr., Obesity is associated with macrophage accumulation in adipose tissue. J Clin Invest 112 (2003) 1796-808.

[5] H. Tilg, and A.R. Moschen, Adipocytokines: mediators linking adipose tissue, inflammation and immunity. Nature reviews 6 (2006) 772-83.

[6] J.P. Thaler, C.X. Yi, E.A. Schur, S.J. Guyenet, B.H. Hwang, M.O. Dietrich, X. Zhao, D.A. Sarruf, V. Izgur, K.R. Maravilla, H.T. Nguyen, J.D. Fischer, M.E. Matsen, B.E. Wisse, G.J. Morton, T.L. Horvath, D.G. Baskin, M.H. Tschop, and M.W. Schwartz, Obesity is associated with hypothalamic injury in rodents and humans. J Clin Invest 122 (2012) $153-62$.

[7] T. Imai, K. Hieshima, C. Haskell, M. Baba, M. Nagira, M. Nishimura, M. Kakizaki, S. Takagi, H. Nomiyama, T.J. Schall, and O. Yoshie, Identification and molecular characterization of fractalkine receptor CX3CR1, which mediates both leukocyte migration and adhesion. Cell 91 (1997) 521-30.

[8] C. Combadiere, K. Salzwedel, E.D. Smith, H.L. Tiffany, E.A. Berger, and P.M. Murphy, Identification of CX3CR1. A chemotactic receptor for the human CX3C chemokine fractalkine and a fusion coreceptor for HIV-1. J Biol Chem 273 (1998) 23799-804.

[9] A.M. Fong, L.A. Robinson, D.A. Steeber, T.F. Tedder, O. Yoshie, T. Imai, and D.D. Patel, Fractalkine and CX3CR1 mediate a novel mechanism of leukocyte capture, firm adhesion, and activation under physiologic flow. J Exp Med 188 (1998) 1413-9.

[10] R. Shah, C.C. Hinkle, J.F. Ferguson, N.N. Mehta, M. Li, L. Qu, Y. Lu, M.E. Putt, R.S. Ahima, and M.P. Reilly, Fractalkine is a novel human adipochemokine associated with type 2 diabetes. Diabetes 60 (2011) 1512-8.

[11] S. Jung, J. Aliberti, P. Graemmel, M.J. Sunshine, G.W. Kreutzberg, A. Sher, and D.R. Littman, Analysis of fractalkine receptor CX(3)CR1 function by targeted deletion and green fluorescent protein reporter gene insertion. Mol Cell Biol 20 (2000) 4106-14.

[12] S. Collins, T.L. Martin, R.S. Surwit, and J. Robidoux, Genetic vulnerability to diet-induced obesity in the C57BL/6J mouse: physiological and molecular characteristics. Physiol Behav 81 (2004) 243-8.

[13] K.J. Kovacs, and G.B. Makara, Corticosterone and dexamethasone act at different brain sites to inhibit adrenalectomy-induced adrenocorticotropin hypersecretion. Brain Res 474 (1988) 205-10. 
[14] D. Zelena, A. Foldes, Z. Mergl, I. Barna, K.J. Kovacs, and G.B. Makara, Effects of repeated restraint stress on hypothalamo-pituitary-adrenocortical function in vasopressin deficient Brattleboro rats. Brain Res Bull 63 (2004) 521-30.

[15] N. Hariri, and L. Thibault, High-fat diet-induced obesity in animal models. Nutr Res Rev 23 (2010) 270-99.

[16] N. Pecoraro, F. Reyes, F. Gomez, A. Bhargava, and M.F. Dallman, Chronic stress promotes palatable feeding, which reduces signs of stress: feedforward and feedback effects of chronic stress. Endocrinology 145 (2004) 3754-62.

[17] M.T. Foster, J.P. Warne, A.B. Ginsberg, H.F. Horneman, N.C. Pecoraro, S.F. Akana, and M.F. Dallman, Palatable foods, stress, and energy stores sculpt corticotropin-releasing factor, adrenocorticotropin, and corticosterone concentrations after restraint. Endocrinology 150 (2009) 2325-33.

[18] N. Hariri, R. Gougeon, and L. Thibault, A highly saturated fat-rich diet is more obesogenic than diets with lower saturated fat content. Nutr Res 30 (2010) 632-43.

[19] B.P. Sampey, A.M. Vanhoose, H.M. Winfield, A.J. Freemerman, M.J. Muehlbauer, P.T. Fueger, C.B. Newgard, and L. Makowski, Cafeteria diet is a robust model of human metabolic syndrome with liver and adipose inflammation: comparison to high-fat diet. Obesity (Silver Spring) 19 (2011) 1109-17.

[20] M. So, M.P. Gaidhu, B. Maghdoori, and R.B. Ceddia, Analysis of time-dependent adaptations in whole-body energy balance in obesity induced by high-fat diet in rats. Lipids Health Dis 10 (2011) 99.

[21] N.J. Rothwell, and M.J. Stock, Thermogenesis and BAT activity in hypophysectomized rats with and without corticotropin replacement. Am J Physiol 249 (1985) E333-6.

[22] N. Satoh, Y. Ogawa, G. Katsuura, Y. Numata, H. Masuzaki, Y. Yoshimasa, and K. Nakao, Satiety effect and sympathetic activation of leptin are mediated by hypothalamic melanocortin system. Neurosci Lett 249 (1998) 107-10.

[23] A.N. Verty, A.M. Allen, and B.J. Oldfield, The endogenous actions of hypothalamic peptides on brown adipose tissue thermogenesis in the rat. Endocrinology 151 (2010) 4236-46.

[24] M. Egawa, H. Yoshimatsu, and G.A. Bray, Neuropeptide Y suppresses sympathetic activity to interscapular brown adipose tissue in rats. Am J Physiol 260 (1991) R328-34.

[25] Y.C. Shi, J. Lau, Z. Lin, H. Zhang, L. Zhai, G. Sperk, R. Heilbronn, M. Mietzsch, S. Weger, X.F. Huang, R.F. Enriquez, P.A. Baldock, L. Zhang, A. Sainsbury, H. Herzog, and S. Lin, Arcuate NPY controls sympathetic output and BAT function via a relay of tyrosine hydroxylase neurons in the PVN. Cell Metab 17 (2013) 236-48.

[26] D.L. Morris, K.E. Oatmen, T. Wang, J.L. DelProposto, and C.N. Lumeng, CX3CR1 deficiency does not influence trafficking of adipose tissue macrophages in mice with dietinduced obesity. Obesity (Silver Spring) 20 (2012) 1189-99.

[27] Y.S. Lee, H. Morinaga, J.J. Kim, W. Lagakos, S. Taylor, M. Keshwani, G. Perkins, H. Dong, A.G. Kayali, I.R. Sweet, and J. Olefsky, The fractalkine/CX3CR1 system regulates beta cell function and insulin secretion. Cell 153 (2013) 413-25.

[28] M.F. Dallman, N. Pecoraro, S.F. Akana, S.E. La Fleur, F. Gomez, H. Houshyar, M.E. Bell, S. Bhatnagar, K.D. Laugero, and S. Manalo, Chronic stress and obesity: a new view of "comfort food". Proc Natl Acad Sci U S A 100 (2003) 11696-701. 
[29] H.E. Auvinen, J.A. Romijn, N.R. Biermasz, L.M. Havekes, J.W. Smit, P.C. Rensen, and A.M. Pereira, Effects of high fat diet on the Basal activity of the hypothalamus-pituitaryadrenal axis in mice: a systematic review. Horm Metab Res 43 (2011) 899-906.

[30] E.E. Kershaw, N.M. Morton, H. Dhillon, L. Ramage, J.R. Seckl, and J.S. Flier, Adipocytespecific glucocorticoid inactivation protects against diet-induced obesity. Diabetes 54 (2005) 1023-31.

[31] T.Y. Man, Z. Michailidou, A. Gokcel, L. Ramage, K.E. Chapman, C.J. Kenyon, J.R. Seckl, and N.M. Morton, Dietary manipulation reveals an unexpected inverse relationship between fat mass and adipose 11 beta-hydroxysteroid dehydrogenase type 1 . Am J Physiol Endocrinol Metab 300 (2011) E1076-84.

[32] M.S. Cooper, and P.M. Stewart, 11Beta-hydroxysteroid dehydrogenase type 1 and its role in the hypothalamus-pituitary-adrenal axis, metabolic syndrome, and inflammation. J Clin Endocrinol Metab 94 (2009) 4645-54.

[33] C.N. Lumeng, S.M. Deyoung, J.L. Bodzin, and A.R. Saltiel, Increased inflammatory properties of adipose tissue macrophages recruited during diet-induced obesity. Diabetes 56 (2007) 16-23.

[34] B.K. Surmi, and A.H. Hasty, The role of chemokines in recruitment of immune cells to the artery wall and adipose tissue. Vascul Pharmacol 52 (2010) 27-36.

[35] P. Lesnik, C.A. Haskell, and I.F. Charo, Decreased atherosclerosis in CX3CR1-/- mice reveals a role for fractalkine in atherogenesis. J Clin Invest 111 (2003) 333-40.

[36] P. Liu, Y.R. Yu, J.A. Spencer, A.E. Johnson, C.T. Vallanat, A.M. Fong, C. Patterson, and D.D. Patel, CX3CR1 deficiency impairs dendritic cell accumulation in arterial intima and reduces atherosclerotic burden. Arterioscler Thromb Vasc Biol 28 (2008) 243-50.

[37] N. Saederup, L. Chan, S.A. Lira, and I.F. Charo, Fractalkine deficiency markedly reduces macrophage accumulation and atherosclerotic lesion formation in CCR2-/- mice: evidence for independent chemokine functions in atherogenesis. Circulation 117 (2008) $1642-8$.

[38] R. Stienstra, J.A. van Diepen, C.J. Tack, M.H. Zaki, F.L. van de Veerdonk, D. Perera, G.A. Neale, G.J. Hooiveld, A. Hijmans, I. Vroegrijk, S. van den Berg, J. Romijn, P.C. Rensen, L.A. Joosten, M.G. Netea, and T.D. Kanneganti, Inflammasome is a central player in the induction of obesity and insulin resistance. Proc Natl Acad Sci U S A 108 15324-9.

[39] B. Vandanmagsar, Y.H. Youm, A. Ravussin, J.E. Galgani, K. Stadler, R.L. Mynatt, E. Ravussin, J.M. Stephens, and V.D. Dixit, The NLRP3 inflammasome instigates obesityinduced inflammation and insulin resistance. Nat Med 17 (2011) 179-88.

[40] C.N. Lumeng, J.L. Bodzin, and A.R. Saltiel, Obesity induces a phenotypic switch in adipose tissue macrophage polarization. J.ClinInvest 117 (2007) 175-84.

[41] C.E. Juge-Aubry, E. Somm, A. Pernin, N. Alizadeh, V. Giusti, J.M. Dayer, and C.A. Meier, Adipose tissue is a regulated source of interleukin-10. Cytokine 29 (2005) 270-4.

[42] R. Stienstra, C. Duval, S. Keshtkar, J. van der Laak, S. Kersten, and M. Muller, Peroxisome proliferator-activated receptor gamma activation promotes infiltration of alternatively activated macrophages into adipose tissue. J Biol Chem 283 (2008) 22620-7.

[43] A. Denes, C. Drake, J. Stordy, J. Chamberlain, B.W. McColl, H. Gram, D. Crossman, S. Francis, S.M. Allan, and N.J. Rothwell, Interleukin-1 mediates neuroinflammatory changes associated with diet-induced atherosclerosis. J Am Heart Assoc 1 (2012) e002006. 


\section{FIGURE LEGENDS}

Figure. 1. Fractalkine receptor deficient mice gain less weight on fat enriched diet

(A) Weight gain in control (Cx3CR1 +/gfp) and fractalkine receptor deficient (Cx3CR1 gfp/gfp) mice during 10 weeks of normal (ND)- or fat enriched diet (FatED). Mean \pm SEM values, * $\mathrm{p}<0.05$ vs. normal diet (ND), \# p<0.05 vs. gfp/gfp (Newman-Keuls post-hoc comparison).

(B) Average food consumption of animals kept on normal (ND)- or fat enriched (FatED) diet. Mean \pm SEM values, $* * \mathrm{p}<0.01, * * * \mathrm{p}<0.001$ vs. normal diet (ND) (Newman-Keuls post-hoc comparison).

(C) Average energy intake of mice on normal (ND)- or fat enriched (FatED) diet. *** $\mathrm{p}<0.0001$ vs. normal diet (ND) (Newman-Keuls post-hoc comparison).

Figure. 2. Effect of fat-enriched diet (FatED) on selected tissues

Changes in epididymal white adipose tissue (EWAT), interscapular brown adipose tissue (IBAT), thymus and liver weights at the end of 10 weeks diet. (Normalized organ weights (organ weight/body weight) are expressed as percentage of corresponding values of +/gfp mice kept on normal diet $(\mathrm{ND})=100 \%$ )

Mean \pm SEM values, * $\mathrm{p}<0.05, * * \mathrm{p}<0.01, * * * \mathrm{p}<0.001$ vs. ND, \# $\mathrm{p}<0.05$, \#\#\# p<0.001 vs gfp/gfp (Newman-Keuls post-hoc comparison).

Figure 3. Effects of fat enriched diet on glucose homeostasis

(A) Mice on fat enriched diet (FatED) have higher basal blood glucose levels than those on normal diet. ** $\mathrm{p}<0.01$ between ND and FatED groups (Factorial ANOVA). After overnight fasting blood glucose levels decrease in all groups compared to fed animals $++\mathrm{p}<0.01,+++$ $\mathrm{p}<0.001$ vs. blood glucose level (Student's t-test) and there is no difference in baseline blood glucose levels in fasted mice.

(B) Blood glucose levels in response to glucose load during glucose tolerance test. Cx3CR1 gfp/gfp mice kept on fat-enriched diet did not develop glucose intolerance $* \mathrm{p}<0.05$ vs. ND, \# $\mathrm{p}<0.05$ vs. gfp/gfp (Newman-Keuls post-hoc comparison).

Figure 4. Effect of fat-enriched diet on the activity of the hypothalamo-pituitary-adrenocortical $\underline{\text { axis }}$

A. Mean \pm SEM values of relative $\mathrm{CRH}$ mRNA levels in the hypothalamus B. plasma ACTHlevels $* \mathrm{p}<0.05$ between ND and FatED groups (diet effect). C.plasma corticosterone levels * $\mathrm{p}<0.05$ between gfp/gfp and +/gfp groups (genotype effect).

Figure. 5. Fractalkine receptor deficiency is associated with reduced macrophage numbers in the white adipose tissue. 
Dot plots showing different fat macrophage populations as identified by flow cytometry using mouse specific PE-labelled F4/80 and APC-labelled MHCII antibodies. Quantitative analysis revealed significantly more macrophages in epididymal fat pads of Cx3CR1 +/- mice, compared to Cx3CR 1 -/- animals (two way ANOVA, P<0.01). F4/80+ MHCIIhigh cells (P4 gate), but not F4/80+ MHCIlint cells (P3 gate) or F4/80+(low) MHCII- cells (P2 gate) are significantly descreased in response to fractalkine receptor deficiency. ${ }^{*} \mathrm{P}<0.05$, two way ANOVA, followed by Sidak's multiple comparison test, $\mathrm{n}=3$.

Figure 6. Effect of fat-enriched diet on different fat depots

(A) Mean \pm SEM values of adipose cell areas measured in epididymal (EWAT) and subcutaneous (SWAT) samples of Cx3CR1 gfp/gfp, and +/gfp animals kept on normal or FatED. $* * * p<0.0001$ vs. ND, \#\#\# p<0.001 vs. gfp/gfp (Newman-Keuls post-hoc comparison).

(B-E) Representative histological images of hematoxylin-eosin stained EWAT sections from Cx3CR1 gfp/gfp mice on normal diet (B) or FatED (C) and +/gfp mice kept on normal- (D) or FatED (E).

Figure 7. Recruitment of leukocytes into the white adipose tissue in mice kept on fat enriched $\underline{\text { diet }}$

A. Mean \pm SEM values of leukocyte/adipocyte ratio in Cx3CR1 +/gfp and gfp/gfp mice kept on normal or fat-enriched diet. * significantly different $(\mathrm{p}=0.03)$ from $+/$ gfp mice on normal diet; \# $\mathrm{p}=0,04$ compared to gfp/gfp mice on fatED.

B. and C. Photomicrographs of hematoxylin-eosin stained sections from epididymal white fat depots of +/gfp mice fatED (B) or on normal diet (C) for 10 weeks. Note the enlarged adipocytes and crown-like aggregation of leukocytes around adipocytes in FatED +/gfp mice (B).

Fig. 8. Effect of fat-enriched diet on various markers in the visceral fat

Mean \pm SEM values for relative mRNA levels in EWAT $* \mathrm{p}<0.05, * * \mathrm{p}<0.01, * * *<0.0001 \mathrm{vs}$. $\mathrm{ND}, \# \mathrm{p}<0.05, \# \#<0.01$, \#\#\#<0.001 vs. gfp/gfp (Newman-Keuls post-hoc comparison). (A) Normalized gfp mRNA levels; chemokines: (B) FKN, (C) MCP-1; (D) 11bHSD1; M1 markers: (E) IL-1a, (F) IL-1b, (G) IL-6, (H) TNFa; (K) NALP3; M2 markers: (I) IL-10, (J) Arg1.

Fig.9. IL-6 is elevated in the liver of Cx3CR1 +/gfp but not in Cx3CR1 gfp/gfp animals 10 weeks after fat enriched diet

Mean \pm SEM values relative mRNA levels in liver. $* * p<0.01$ vs. ND, \#\# $p<0.01$ vs gfp/gfp (Newman-Keuls post-hoc comparison). M1 markers: (A) IL-1a, (B) IL-1b, (C) IL-6, (D) TNFa; (E) MCP-1 chemokine; (F) normalized gfp mRNA levels,. 
Fig.10. Expression of select immune markers in the hypothalamus of fractalkine receptor deficient (Cx3CR1 gfp/gfp) and control (CX3CR1 +/gfp) mice following 10 week fat enriched $\underline{\text { diet }}$

Relative mRNA levels in hypothalamus. $* *$ p $<0.01$ vs. ND, \#\#\# p<0.001 vs. gfp/gfp (NewmanKeuls post-hoc comparison) (A)Normalized gfp mRNA levels; (B) MCP-1 chemokine, (C) orexigenic NPY mRNA levels, (D) anorexigenic POMC mRNA levels; proinflammatory cytokines: (E) IL-1a, (F) IL-1b, (G) IL-6, (H) TNFa

Figure 11. Plasma IL-1 $\beta$ is upregulated in response to fat enriched diet in $+/ g f p$, but not in gfp/gfp mice

IL-1 $\beta$, IL- $1 \alpha$ and IL-6 levels were measured from plasma samples with ELISA after 10 weeks of fat enriched diet (FatED) or normal diet (ND). $* * \mathrm{P}<0.01$, two-way ANOVA followed by Sidak's post hoc test. 